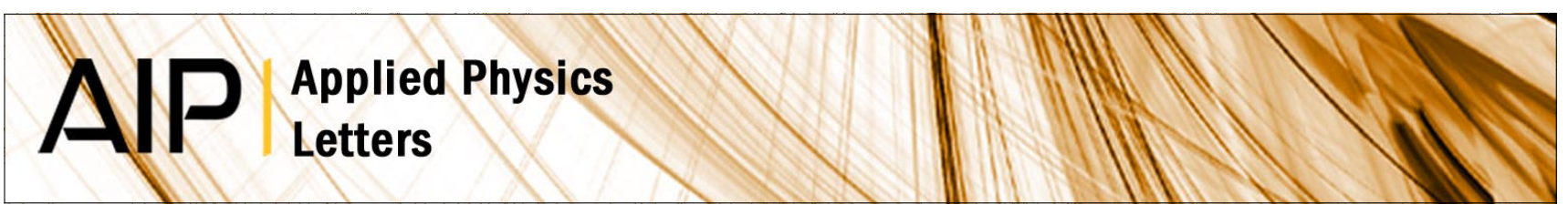

\title{
Effects of random link removal on the photonic band gaps of honeycomb networks
}

Marian Florescu, Salvatore Torquato, and Paul J. Steinhardt

Citation: Appl. Phys. Lett. 97, 201103 (2010); doi: 10.1063/1.3505322

View online: http://dx.doi.org/10.1063/1.3505322

View Table of Contents: http://apl.aip.org/resource/1/APPLAB/v97/i20

Published by the American Institute of Physics.

\section{Related Articles}

Interplay of index contrast with periodicity in polymer photonic crystals Appl. Phys. Lett. 99, 261913 (2011)

Off-resonant coupling between a single quantum dot and a nanobeam photonic crystal cavity Appl. Phys. Lett. 99, 251907 (2011)

Phase-locking regimes of photonic crystal nanocavity laser arrays

Appl. Phys. Lett. 99, 251104 (2011)

Field confinement and quality factor of the multilayer cavity resonators

J. Appl. Phys. 110, 114519 (2011)

Slow light in one dimensional metallic-dielectric photonic crystals due to sign change of the effective dielectric constant

Appl. Phys. Lett. 99, 221916 (2011)

\section{Additional information on Appl. Phys. Lett.}

Journal Homepage: http://apl.aip.org/

Journal Information: http://apl.aip.org/about/about_the_journal

Top downloads: http://apl.aip.org/features/most_downloaded

Information for Authors: http://apl.aip.org/authors

\section{ADVERTISEMENT}

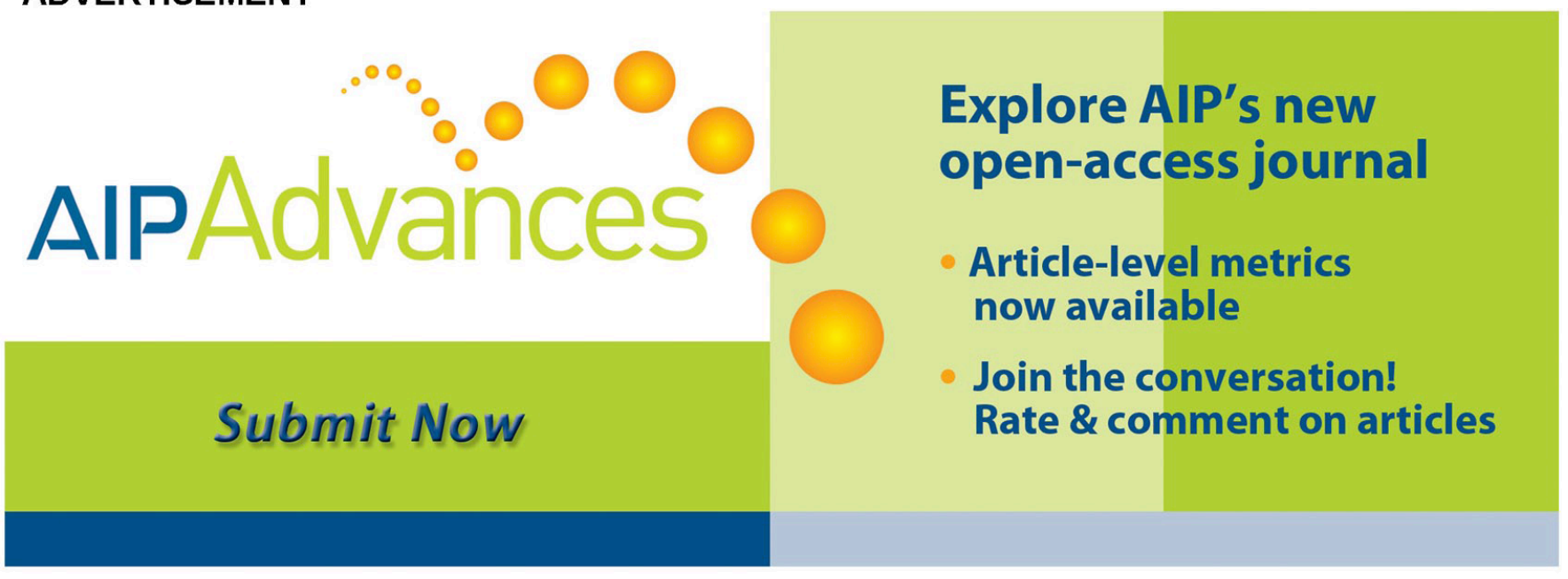




\title{
Effects of random link removal on the photonic band gaps of honeycomb networks
}

\author{
Marian Florescu, ${ }^{1, a)}$ Salvatore Torquato, ${ }^{1,2,3,4}$ and Paul J. Steinhardt ${ }^{1,3}$ \\ ${ }^{1}$ Department of Physics, Princeton University, Princeton, New Jersey 08544, USA \\ ${ }^{2}$ Department of Chemistry, Princeton University, Princeton, New Jersey 08544, USA \\ ${ }^{3}$ Princeton Center for Theoretical Science, Princeton University, Princeton, New Jersey 08544, USA \\ ${ }^{4}$ Princeton Institute for the Science and Technology of Materials, Princeton University, Princeton, New Jersey \\ 08544, USA
}

(Received 26 June 2010; accepted 4 October 2010; published online 16 November 2010)

\begin{abstract}
We explore the effects of random link removal on the photonic band gaps of honeycomb networks. Missing or incomplete links are expected to be common in practical realizations of this class of connected network structures due to unavoidable flaws in the fabrication process. We focus on the collapse of the photonic band gap due to the defects induced by the link removal. We show that the photonic band gap is quite robust against this type of random decimation and survives even when almost 58\% of the network links are removed. (C) 2010 American Institute of Physics.

[doi:10.1063/1.3505322]
\end{abstract}

Photonic crystals, and, in particular, photonic band gap (PBG) materials, ${ }^{1,2}$ constitute a class of dielectric materials, in which the basic electromagnetic interaction is controllably altered over certain frequencies and length scales. ${ }^{3,4}$ The ability to tailor the photonic density of states in a prescribed manner and to engineer the symmetry properties of the electromagnetic field inside a PBG material enables the design of materials and devices that control the flow and the emission and absorption properties of electromagnetic radiation. ${ }^{5-8}$ Most of the photonic crystals fabricated to date possess intrinsic disorder, including size variation among the scattering centers, roughness of the dielectric walls, point defects, lattice dislocation, and stacking defaults. ${ }^{9,10}$ The presence of disorder results in defects modes that can fill the PBG region partially or completely and alter significantly the photonic properties of the dielectric crystals.

In this paper we focus on the effect of random link removal on the properties of photonic gaps of honeycomb networks. It is well established that two-dimensional dielectric structures based on connected network architectures favor the formation of large PBGs for transverse electric (TE) polarized radiation. ${ }^{11-13}$ The largest known TE PBG is achieved in a dielectric structure obtained by decorating the links of a honeycomb network lattice with dielectric walls of a certain thickness ${ }^{14}$ (also depicted in the inset of Fig. 1).

We consider a special type of disorder produced by the random removal of links from a honeycomb connected network. This type of disorder is expected to be common in practical realizations of this class of connected network structures due to unavoidable flaws in the fabrication process. The major goals of this study are to explore the robustness of the PBG against random removal of links and to establish a relationship between the topology of defect complexes (resulting from the removal of multiple links) and the size of the band gap.

The class of photonic structures considered in this study are generated by decorating the sides of a two-dimensional honeycomb tessellation with dielectric walls of dielectric

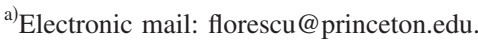

constant $\epsilon=11.56$ (corresponding to $\mathrm{Si}$ in the infrared region) and thickness $w / a=0.94$, where $a$ is the honeycomb lattice constant, corresponding to the largest known TE PBG: $52.63 \%$ (see Fig. 1).

To evaluate the influence of the link disorder on the band gap properties, we construct a finite domain of the honeycomb dielectric network and define a statistically independent probability, $p$, for the removal of the dielectric links (i.e., Bernoulli percolation ${ }^{15,16}$ ) that connect the vertices of the honeycomb network. For each $p$, a statistical ensemble consisting of 1500 configurations is generated, and the photonic band structure is calculated along a closed contour around the Brillouin zone [as in Figs. 1, 3(a), and 4(a)] for each member of the ensemble. In addition, we have checked that gap size by calculating the band structure for 100 k-points randomly distributed in the first Brillouin zone. Due to computational limitations, the domain considered consists of $8 \times 8$ unit cells. In Fig. 2, we plot the maximum, minimum, and average PBG in a given ensemble of randomly generated structures as a function of the number of links

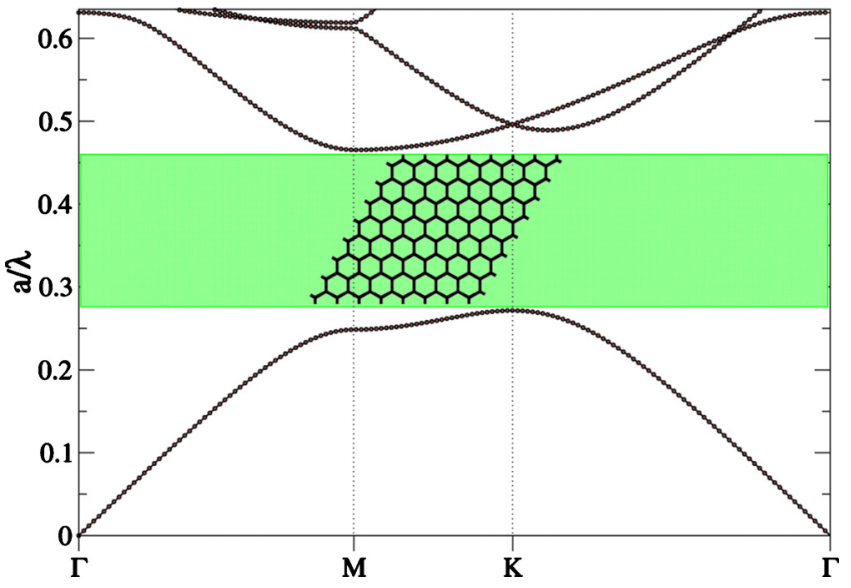

FIG. 1. (Color online) Band structure for the optimal unperturbed TE PBG structure consisting of dielectric walls with dielectric constant $\epsilon=11.56$ thickness $w / a=0.94$ (shown as an inset), which presents a TE PBG of $\Delta \omega / \omega_{C}=52.63 \%$, the largest known PBG in two-dimensional photonic crystals for this dielectric constant contrast ratio. 


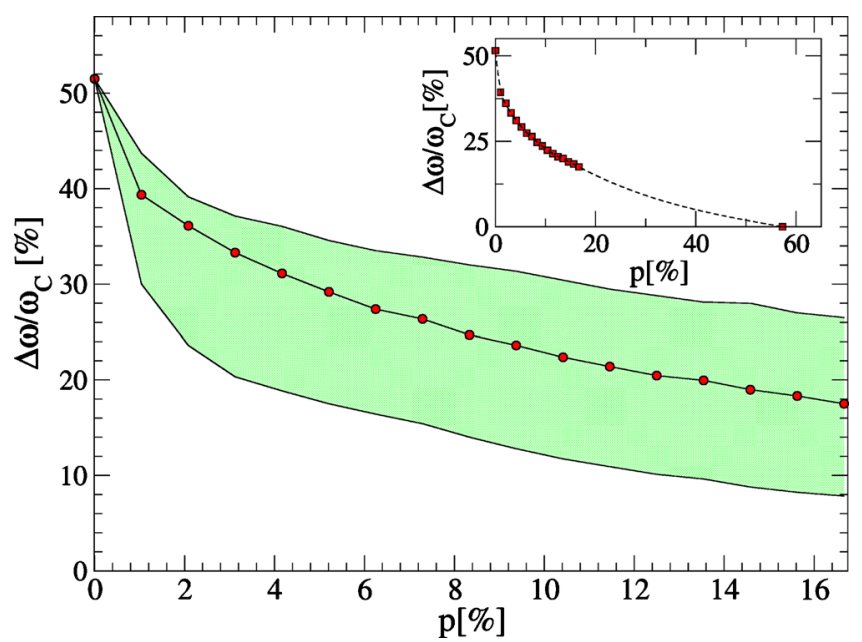

FIG. 2. (Color online) The small filled circles represent the PBG size as a function of the percentage of randomly removed links, as obtained by averaging over a statistical ensemble of 1500 configurations. The upper and lower lines represent the maximum and minimum value among the ensemble. The inset shows the extrapolation of the average PBG to percentages large enough to close the photonic band gap.

removed. We note that while full connectivity is associated with the largest PBG structures, ${ }^{17}$ the band gap is very resilient against line removal; even after the random removal of $p=16.7 \%$ of all the links, the structures present a sizable

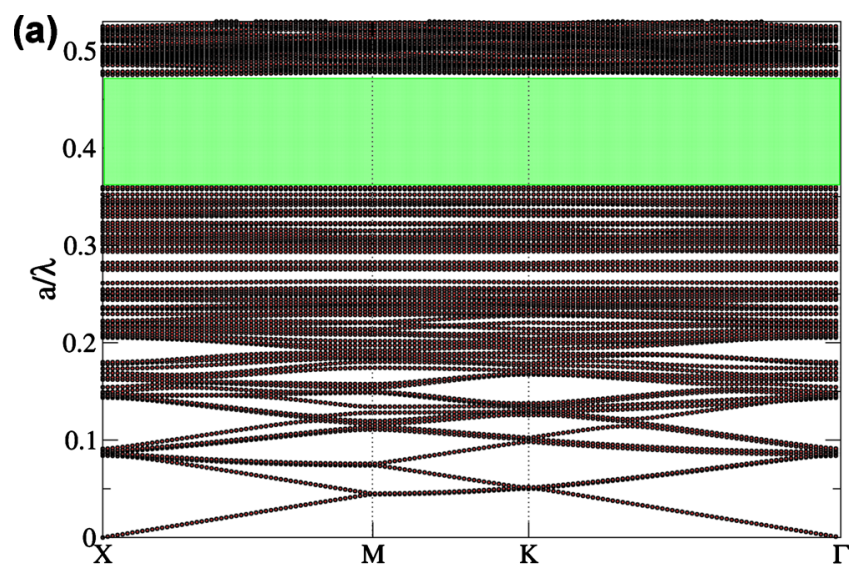

(b)

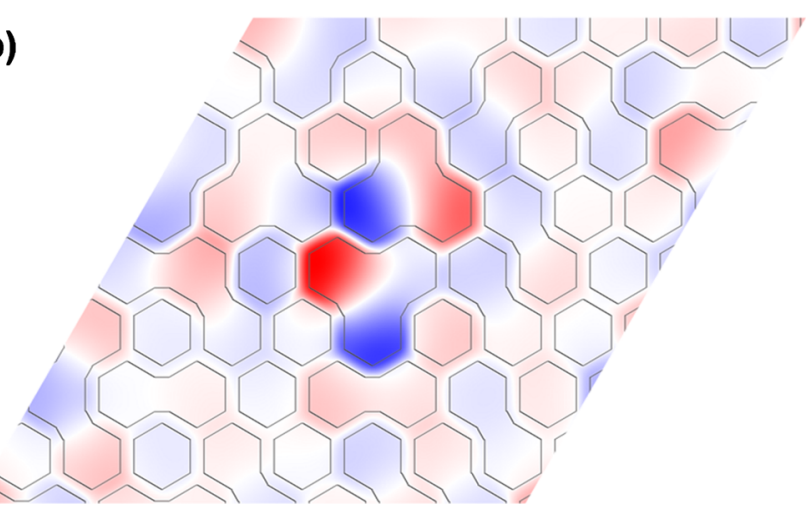

FIG. 3. (Color online) The connected network photonic structure with the largest TE PBG for $p=16.7 \%$ (selected from 1500 randomly generated configurations). (a) The photonic band structure for dielectric constant contrast $\epsilon=11.56$ and wall thickness $w / a=0.94$, displaying a PBG with $\Delta \omega / \omega_{C}$ $=27.78 \%$ (shaded region). (b) The magnetic field distribution for the mode at the lower edge of the PBG (the black lines show the contour of dielectric material distribution).

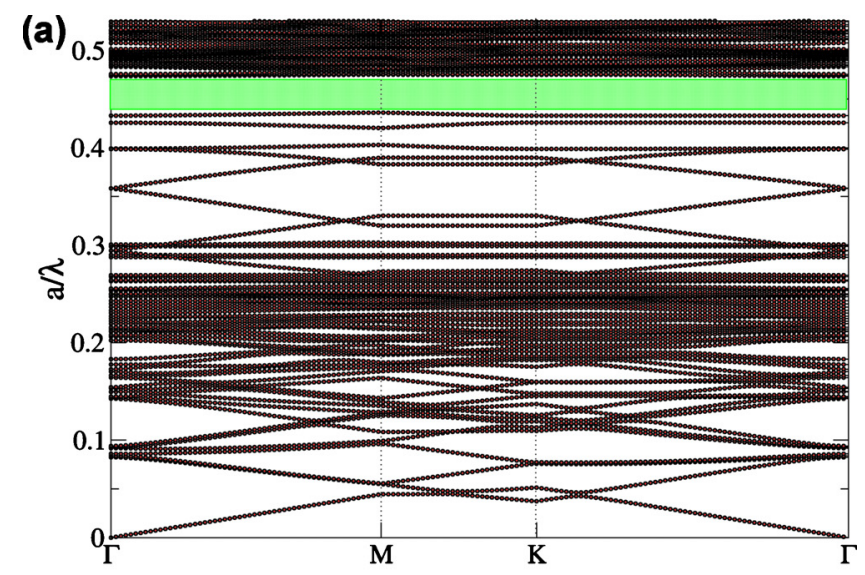

(b)

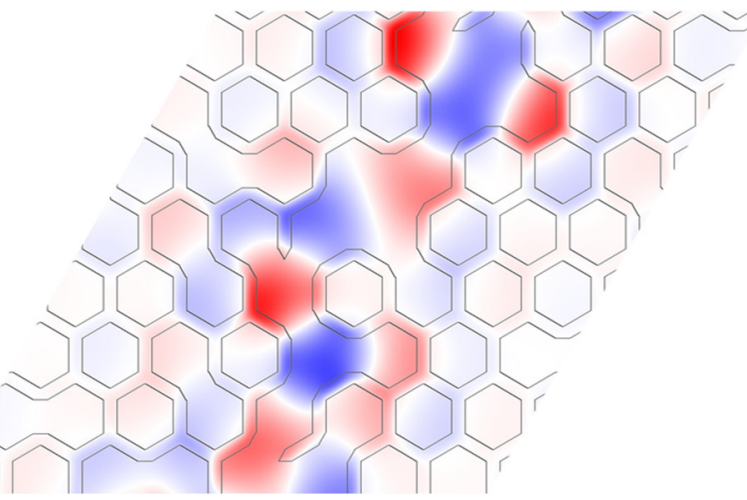

FIG. 4. (Color online) The connected network photonic structure presenting the smallest TE PBG for $p=16.7 \%$ (selected from 1500 randomly generated configurations). (a) The photonic band structure for dielectric constant contrast $\epsilon=11.56$ and wall thickness $w / a=0.94$, displaying a PBG $\Delta \omega / \omega_{C}$ $=7.95 \%$. (b) The magnetic field distribution for the mode corresponding to the lower edge of the PBG (the black lines show the contour of dielectric material distribution).

average band gap of $\Delta \omega / \omega_{C}=17 \%$ (here $\Delta \omega$ is the PBG size and $\omega_{C}$ the gap central frequency). Moreover, the minimal PBG configuration still retains a gap of about $8 \%$, while the maximal PBG configurations display sizable band gaps of about $28 \%$.

We have analyzed different types of defects generated by the link removal and their influence on the band gap. In general, the link removal results in a lowering of the local dielectric constant, which, as expected, generates an increase in the frequency of the photonic modes around the lower edge of the PBG. These modes ascend inside the PBG and subsequently the PBG size is reduced. As the density of the removed links increases, the unoccupied links may cluster together to generate larger defect complexes. Isolated clusters containing a small number of missing links generate cavitylike defects. Cavitylike defects induce photonic modes with a high degree of localization and low dispersion, and generate defect bands with small spectral bandwidth.

In Fig. 3 a we plot the band structure for the configuration that displays the largest gap $\left(\Delta \omega / \omega_{C}=27.78 \%\right)$ in a structure with $p=16.7 \%$. We note that the modes shift to inside the PBG due to the presence of material defects present minimal dispersion. The corresponding dielectric structure [the black-line contour in Fig. 3(b)] and the magnetic field distribution of the photonic mode defining the lower edge of the PBG confirm that most of the defect modes 
are confined around the random cavitylike defects in the structure.

A second type of defect is a waveguidelike complex, which occurs when removed links concatenate to create a defect-cluster spanning the entire structure. In this case, the defect modes are associated with propagation along the waveguide channel, thus localized only transverse to the waveguide. As a result, they display stronger dispersion and have a more deleterious effect on the width of the PBG.

In Fig. 4(a), we plot the band structure for the configuration that achieves the minimal PBG $\left(\Delta \omega / \omega_{C}=7.96 \%\right)$ among structures with $p=16.7 \%$. Here we note that among the modes that partially fill the $\mathrm{PBG}$ region, there are modes showing considerable dispersion. As shown in Fig. 4(b), the unoccupied links form a waveguide channel (the dielectric profile is shown by the black-line contour), and the mode at the lower edge of the PBG has the magnetic field rather tightly confined inside the waveguide.

To summarize, we have analyzed the effects of link disorder on the TE band gaps in honeycomb connected network photonic structures. We have demonstrated that the PBG is robust against this type of disorder and have identified the relevant defect topologies. Removing links of dielectric material introduces zero-dimensional (cavities) or onedimensional (waveguide channels) defects, and, depending on their spectral location and dispersion, the PBG size is reduced as $p$ increases. At some threshold value of $p$, the PBG vanishes. Thus, our study can be regarded to be a photonic percolation problem. We define the photonic percolation threshold as the value $p=p_{c}$ at which the PBG first vanishes in the infinite-system limit. For $p \geq p_{c}$, the PBG is identically zero. Computational requirements make difficult a precise determination of the photonic percolation threshold but we have performed an extrapolation of our results (shown in the inset of Fig. 2), which indicates that the PBG vanishes if, on average, $p_{c}=57.6 \%$ of the links are removed. Further band structure calculations confirm that, if $57.6 \%$ of the connected network links are randomly removed, the av- erage PBG collapses. This photonic-percolation-threshold value should be contrasted with that of Bernoulli nearestneighbor bond percolation on a honeycomb network for which $p_{c}=1-2 \sin \pi / 18 \approx 65.28 \%$. The fact that the values of $p_{c}$ are not identical is not surprising because they depend in detail on different physical characteristics. Other percolation phenomena with yet different values $p_{c}$ include elasticity percolation $^{16,18}$ and quantum percolation. ${ }^{19}$

This work was supported by the MRSEC Program of the National Science Foundation under Awards DMR0820341 and ECCS-1041083.

${ }^{1}$ S. John, Phys. Rev. Lett. 58, 2486 (1987).

${ }^{2}$ E. Yablonovitch, Phys. Rev. Lett. 58, 2059 (1987).

${ }^{3}$ J. D. Joannopoulos, R. D. Meade, and J. N. Winn, Photonic Crystals (Princeton University Press, Princeton, NJ, 1995).

${ }^{4}$ K. Busch, G. von Freymann, S. Linden, S. F. Mingaleev, L. Tkeshelashvili, and M. Wegener, Phys. Rep. 444, 101 (2007).

${ }^{5}$ S. John and T. Quang, Phys. Rev. A 50, 1764 (1994).

${ }^{6}$ S. John and T. Quang, Phys. Rev. Lett. 78, 1888 (1997).

${ }^{7}$ O. Painter, R. K. Lee, A. Scherer, A. Yariv, J. D. O'Brien, P. D. Dapkus, and I. Kim, Science 284, 1819 (1999).

${ }^{8}$ M. Florescu and S. John, Phys. Rev. A 69, 053810 (2004).

${ }^{9}$ Y. A. Vlasov, V. N. Astratov, A. V. Baryshev, A. A. Kaplyanskii, O. Z. Karimov, and M. F. Limonov, Phys. Rev. E 61, 5784 (2000).

${ }^{10}$ T. F. Krauss, R. M. De La Rue, and S. Brand, Nature (London) 383, 699 (1996)

${ }^{11}$ O. Sigmund and K. Hougaard, Phys. Rev. Lett. 100, 153904 (2008).

${ }^{12}$ M. Florescu, S. Torquato, and P. J. Steinhardt, Phys. Rev. B 80, 155112 (2009).

${ }^{13}$ M. Florescu, S. Torquato, and P. J. Steinhardt, Proc. Natl. Acad. Sci. U.S.A. 106, 20658 (2009).

${ }^{14}$ H. Fu, Y. Chen, R. Chen, and C. Chang, Opt. Express 13, 7854 (2005).

${ }^{15}$ L. Berlyand, M. D. Rintoul, and S. Torquato, J. Stat. Phys. 91, 603 (1998).

${ }^{16} \mathrm{~S}$. Torquato, Random Heterogeneous Materials: Microstructure and Macroscopic Properties (Springer, New York, 2002).

${ }^{17}$ S. Fan, P. R. Villeneuve, R. D. Meade, and J. D. Joannopoulos, J. Appl. Phys. 78, 1415 (1995).

${ }^{18} \mathrm{M}$. Sahimi, Applications of Percolation Theory (Taylor and Francis, London, 1994)

${ }^{19}$ C. M. Soukoulis and G. S. Grest, Phys. Rev. B 44, 4685 (1991). 